\title{
Self Fourier functions and fractional Fourier transforms
}

\author{
David Mendlovic \\ Tel-Aviv University, Faculty of Engineering, 69978 Tel-Aviv, Israel
}

Haldun M. Ozaktas

Electrical Engineering, Bilkent University, 06533 Bilkent, Ankara, Turkey

and

\author{
Adolf W. Lohmann \\ Angewandte Optik, Erlangen University, D-91058 Erlangen, Germany
}

Received 4 May 1993; revised manuscript received 25 August 1993

\begin{abstract}
Self Fourier functions and fractional Fourier transforms are two concepts that have been discussed recently. Investigated is the combination of these two concepts: self fractional Fourier functions and the fractional Fourier transform of a self Fourier function.
\end{abstract}

The Fourier transform is perhaps the most important analytical tool in wave optics. Hence Fourier-related concepts are likely to have an impact on optics. We will now recall two novel concepts and then show how they are interrelated. The two functions $f$ and $\tilde{f}$ are a Fourier pair if

$\tilde{f}(\nu)=\int f(x) \exp (-2 \pi \mathrm{i} \nu x) \mathrm{d} x$,

$f(x)=\int \tilde{f}(\nu) \exp (2 \pi \mathrm{i} \nu x) \mathrm{d} \nu$.

A self Fourier function (SFF) is a function whose Fourier transform is identical to itself, i.e.

$\tilde{f}(\nu)=f(\nu)$.

Caola [1] showed how to construct a SFF $F(x)$ from any arbitrary transformable function $g(x)$,

$F(x)=g(x)+\tilde{g}(x)+g(-x)+\tilde{g}(-x)$.

It was shown [2] that any SFF can be decomposed in this manner. Thus, $F(x)$ is an SFF if, and only if, it can be expressed as the sum of four functions in the form of the above equation. Additional SFF studies are reported in refs. [3-5].

Another issue that has been recently investigated is the fractional Fourier transform [6-9]. Two distinct definitions of the fractional Fourier transform have been given. In the first one [6-8], the fractional Fourier transform was defined physically, based on the propagation in quadratic graded index (GRIN) media.

The second definition is based on Wigner distribution functions (WDF) [9]. Here the fractional Fourier transform is calculated by finding the WDF of the input image, rotating it by an angle $\alpha=a \pi / 2$, and performing the inverse Wigner transform.

It was shown [10] that both definitions of the fractional Fourier transform are equivalent.

In this communication, we first discuss fractional Fourier transforms of SFFs and then discuss self fractional Fourier functions (SFFF's). Assuming an SFF $F(x)$ with a generating function $g(x)$, one can rewrite eq. (4) as

$$
F(x)=\mathscr{F}^{0} g(x)+\mathscr{F}^{1} g(x)+\mathscr{F}^{2} g(x)+\mathscr{F}^{3} g(x) .
$$


Now, the fractional Fourier transform of $F(x)$ of or$\operatorname{der} a$ is

$$
\begin{aligned}
\mathscr{F}^{a} F(x)=\mathscr{F}^{a}\left[\mathscr{F}^{0} g(x)\right. & +\mathscr{F}^{1} g(x) \\
& \left.+\mathscr{F}^{2} g(x)+\mathscr{F}^{3} g(x)\right] .
\end{aligned}
$$

Due to the additivity property of the fractional Fourier transform, the last equation becomes

$$
\begin{gathered}
\mathscr{F}^{a} F(x)=\mathscr{F}^{a} g(x)+\mathscr{F}^{1+a} g(x) \\
+\mathscr{F}^{2}+a g(x)+\mathscr{F}^{3+a} g(x) \\
=\mathscr{F}^{0} \mathscr{F}^{a} g(x)+\mathscr{F}^{1} \mathscr{F}^{a} g(x) \\
+\mathscr{F}^{2} \mathscr{F} a g(x)+\mathscr{F}^{3} \mathscr{F}^{a} g(x) .
\end{gathered}
$$

Defining $G(x)$ through the equation

$$
G(x)=F^{a} g(x)
$$

we obtain

$$
\mathscr{F}^{a} F(x)=\mathscr{F}^{0} G(x)+\mathscr{F}^{1} G(x)+\mathscr{F}^{2} G(x)+\mathscr{F}^{3} G(x),
$$

$$
\mathscr{F}^{a} F(x)=G(x)+\tilde{G}(x)+G(-x)+\tilde{G}(-x) .
$$

This means that the fractional Fourier transform of an SFF can be expressed in terms of the fractional Fourier transform of its generating function $g(x)$. Since this equation is of the same form as eq. (4), we conclude that the fractional Fourier transform of an SFF is also an SFF.

Now we address the question of how to generate a self fractional Fourier function (SFFF) in other words, the eigen function of the fractional Fourier operation. Assuming a generating function $g(x)$ and a rational fractional Fourier transform order $a=$ $N / M$ (where $N$ and $M$ are integers), the following function $F(x)$ is the same as its $a=N / M$ th transform:

$$
\begin{aligned}
F(x)=\left[\mathscr{F}^{0}+\mathscr{F}^{N / M}+\mathscr{F}^{2 N / M}+\ldots\right. \\
\\
\left.+\mathscr{F}^{(k-1) N / M}\right] g(x) .
\end{aligned}
$$

$k$ and $L$ are the smallest integers that satisfy

$k N / M=4 L$

for the given $N$ and $m$.

Proof:

$$
\begin{gathered}
\mathscr{F}^{N / M} F(x)=\mathscr{F}^{N / M}\left(\mathscr{F}^{0}+\mathscr{F}^{N / M}+\ldots\right. \\
\left.+\mathscr{F}^{(k-1) N / M}\right) g(x) \\
=\left(\mathscr{F}^{N / M}+\mathscr{F}^{2 N / M}+\ldots+\mathscr{F}^{k N / M}\right) g(x) .
\end{gathered}
$$

By virtue of eq. (12), $\mathscr{F}^{k N / M}=\mathscr{F}^{4 L}$. A well known property of the Fourier transform operator is that $\mathscr{F}^{4 L}=\mathscr{F}^{0}$. Thus

$$
\begin{gathered}
\mathscr{F}^{N / M} F(x)=\left[\mathscr{F}^{N / M}+\mathscr{F}^{2 N / M}+\ldots\right. \\
\left.+\mathscr{F}^{(k-1) N / M}+\mathscr{F}^{0}\right] g(x)=F(x),
\end{gathered}
$$

which shows that $F(x)$ is an SFFF for the order $N / M$.

Equation (11) has $k=4 L M / N$ terms. We can always choose $L=N$ in the worst case, resulting in $k=4 M$ terms in the summation. However, sometimes smaller values of $k$ can also fulfill eq. (12), resulting in fewer terms in the summation.

The function $F(x)$ constructed above is a self fractional Fourier function only for the order $N / M$. It is also possible to construct a SFFF for given $M$, but arbitrary $N$. Such a function is again given by eq. (11), but now we choose $k=4 M$. Through a similar proof as given above, it is possible to show that $\mathscr{F}^{N / M} F(x)=F(x)$ for all values of $N$.

A final remark about the relevance of these transform studies: a reversible transform does neither add nor substract any information. But the transform may emphasize certain signal features which were present but hidden before the signal was transformed. Some remarks of this kind are contained in the references. Other remarks will appear in several papers that are under preparation. For example, experimental results are in ref. [3]. A particular comment by Lipson [5] stipulates the relation between self fractional Fourier functions and "quasi-confocal resonators". These are resonators in which a signal recovers its shape not after one loop but for example after three loops. The resonator distance might be a simple fraction (like 2/3) of the focal length. Although these remarks are not a definitive proof of the relevance of our studies, we are nevertheless optimistic based on the fundamental argument stated in the very first sentence of this paper.

\section{References}

[1] M.J. Caola, J. Phys, A 24 (1991) L 1143.

[2] A.W. Lohmann and D. Mendlovic, J. Opt. Soc. Am. A 9 (1992) 2009.

[3] A.W. Lohmann and D. Mendlovic, Image formation of a self Fourier object, Appl. Optics, to be published. 
[4] G. Cincotti, F. Gori and M. Santarsiero, J. Phys. A 25 (1992) L 1191.

[5] S.G. Lipson and H. Lipson, Optical physics, 2nd Ed. (Cambridge, Cambridge University Press, 1981) p. 191; J. Opt. Soc. Am. A 10 (1993) 2088.

[6] D. Mendlovic and H.M. Ozaktas, J. Opt. Soc. Am. A 10 (1993) 1875.

[7] H.M. Ozaktas and D. Mendlovic, Fractional Fourier transformations and their optical implementation: Part II, J. Opt. Soc. Am. A, to be published.
[8] H.M. Ozaktas and D. Mendlovic, Optics Comm. 101 (1993) 163.

[9] A.W. Lohmann, J. Opt. Soc. Am. A 10 (1993) 2181.

[10] D. Mendlovic, H.M. Ozaktas and A.W. Lohmann, The effect of propagation in graded index media on the Wigner distribution function and the equivalence of two definitions of the fractional Fourier transform, Appl. Optics, to be published. 